\title{
Valediction: descriptions of novel prokaryotic taxa published in Antonie van Leeuwenhoek-change in editorial policy and a signpost to the future?
}

\author{
Iain C. Sutcliffe
}

Published online: 2 August 2019

(C) Springer Nature Switzerland AG 2019

Over the past decade Antonie van Leeuwenhoek has emerged as a significant journal supporting microbial systematics research. In that period the journal has published more than 900 systematics papers and reviews, mainly descriptions of novel prokaryotic species, along with significant papers on yeast taxonomy (for example Kurtzman 2011; Daniel et al. 2014). Importantly it has been central to the work of the journal's Editors to uphold the principles and rules of the international Codes that are crucial to ensuring stable nomenclature of both fungal and prokaryotic taxa (Sutcliffe and Trujillo 2013). Indeed, it is rewarding that a high percentage of the new prokaryotic names 'effectively published' in Antonie van Leeuwenhoek are subsequently validated in the Lists published by the International Journal of Systematic and Evolutionary Microbiology (Oren et al. 2018).

The Editors have strongly encouraged the use of genome sequence data in the description on novel prokaryotic taxa. We have also contributed to the promotion of systematics as a discipline area through the production of two Special Issues based on the 1st and 3rd meetings of Bergey's International Society for Microbial Systematics (Sutcliffe et al. 2012a; Hedlund

I. C. Sutcliffe (两)

Department of Applied Sciences, Faculty of Health and Life Sciences, Northumbria University,

Newcastle upon Tyne, UK

e-mail: iain.sutcliffe@northumbria.ac.uk et al. 2017) and significant reviews on the future development of microbial systematics (for example Sutcliffe et al. 2012b; Oren and Garrity 2014; Vandamme and Peeters 2014). Perhaps most ambitiously, the journal partnered with Systematic and Applied Microbiology to launch the Digital Protologue Database (Rosselló-Móra et al. 2017), "a partial success" which has provided important insights and lessons for those wishing to pick up the baton and take forward the vital task of building an effective database to underpin microbial taxonomy (Rosselló-Móra and Sutcliffe 2019).

However, as highlighted previously (Sutcliffe et al. 2012b; Sutcliffe 2015; Rosselló-Móra and Sutcliffe 2019), it seems unfeasible, given the sheer scale of microbial diversity, that a "one species, one paper" publishing model can be sustained indefinitely, particularly if there is to be a shift towards 'high throughput' approaches to the circumscription and valid naming of prokaryotic taxa. In anticipation of this, it has been decided that there will be a change in editorial policy in Antonie van Leeuwenhoek regarding publication of routine descriptions of novel prokaryotic taxa. Hereon, these will only be considered for peer review where the authors can make a convincing case for demonstrating novel biology and features of interest (and/or ecological insights relevant to the isolation source) in a proposed novel taxon. These may be experimentally demonstrated and/or inferred from genome sequence analysis. Moreover, 
we again emphasise our belief that it is best taxonomic practise to describe novel prokaryotic and fungal taxa based upon multiple strains. We will also continue to encourage the submission of larger scale overarching studies of genera and higher taxa, such as phylogenomic analyses. It is also emphasised that the journal's specialist Editors will continue to welcome submissions describing novel fungal taxa.

In view of the need to change editorial policy regarding descriptions of novel prokaryotic taxa, it is inevitable that the nature and balance of the journal's content will change over time. After a full 10 years at the helm, it therefore seems an appropriate time for me to stand down as Editor-in-Chief. Springer Nature will shortly announce the appointment of a new Editor-inChief and I wish the incoming Editor great success: I am sure they will enjoy the challenges of the role as much as I have.

During my tenure, it has been my great pleasure to work with a dedicated and enthusiastic team of Editors, supported by the excellent staff in the Springer Nature editorial office. I thank all of these people for the considerable time and energy that they have invested in sustaining a journal now midway through its 9th decade and with a bright future!

\section{References}

Daniel HM, Lachance MA, Kurtzman CP (2014) On the reclassification of species assigned to Candida and other anamorphic ascomycetous yeast genera based on phylogenetic circumscription. Antonie van Leeuwenhoek 106:67-84
Hedlund BP, Sutcliffe IC, Trujillo ME (2017) Special Issue: the 3rd meeting of the Bergey's International Society for Microbial Systematics (BISMIS). Antonie Van Leeuwenhoek 110:1245-1246

Kurtzman CP (2011) Phylogeny of the ascomycetous yeasts and the renaming of Pichia anomala to Wickerhamomyces anomalus. Antonie Van Leeuwenhoek 99:13-23

Oren A, Garrity GM (2014) Then and now: a systematic review of the systematics of prokaryotes in the last 80 years. Antonie Van Leeuwenhoek 106:43-56

Oren A, Garrity GM, Parte AC (2018) Why are so many effectively published names of prokaryotic taxa never validated? Int J Syst Evol Microbiol 68:2125-2129

Rosselló-Móra R, Sutcliffe IC (2019) Reflections on the introduction of the digital protologue database-a partial success? Antonie Van Leeuwenhoek 112:141-143

Rosselló-Móra R, Trujillo M, Sutcliffe IC (2017) Introducing a digital protologue: a timely move towards a database-driven systematics of archaea and bacteria. Antonie Van Leeuwenhoek 110:455-456

Sutcliffe IC (2015) Challenging the anthropocentric emphasis on phenotypic testing in prokaryotic species descriptions: rip it up and start again. Front Genet 6:218

Sutcliffe IC, Trujillo ME, Goodfellow M (2012) The BISMiS 2011 Special Issue on prokaryotic systematics, a vital discipline entering a period of transition. Antonie Van Leeuwenhoek 101:1-2

Sutcliffe IC, Trujillo ME, Goodfellow M (2012) A call to arms for systematists: revitalising the purpose and practises underpinning the description of novel microbial taxa. Antonie Van Leeuwenhoek 101:13-20

Sutcliffe IC, Trujillo ME (2013) Publication of descriptions of novel microbial taxa in Antonie van Leeuwenhoek. Antonie Van Leeuwenhoek 103:1-2

Vandamme P, Peeters C (2014) Time to revisit polyphasic taxonomy. Antonie Van Leeuwenhoek 106:57-65

Publisher's Note Springer Nature remains neutral with regard to jurisdictional claims in published maps and institutional affiliations. 